\title{
A CHARACTERIZATION OF DENJOY FLOWS
}

\author{
KONSTANTIN ATHANASSOPOULOS
}

1

An interesting problem in the theory of dynamical systems is to determine the global structure of a flow from properties of characteristic invariant subsets of the phase space such as minimal sets or Poisson stable orbit closures. For flows on manifolds of dimension greater than two, the behaviour of the flow near such sets is far from being well understood. In this note we give a characterization of Denjoy flows on the torus, that is, suspensions of orientation preserving homeomorphisms of the unit circle onto itself with a Cantor minimal set, via conditions referring to the asymptotic behaviour of the orbits near a strictly Poisson stable orbit closure. More precisely, we prove the following.

THEOREM 1.1. A flow $(\mathbb{R}, M, f)$ on a closed 2-manifold $M$ is topologically equivalent to a Denjoy or irrational flow if and only if there is a strictly Poisson stable orbit $C(x)$ and an open invariant neighbourhood $V$ of $\overline{C(x)}$ such that $L^{+}(y)=L^{-}(y)$ for every $y \in V$.

This theorem is first proved in the particular case where the strictly Poisson stable orbit is contained in a non-trivial minimal set, using the description of the flow near non-trivial minimal sets on 2-manifolds given in [2]. The general case is proved using this and a cutting and pasting technique.

Let $(\mathbb{R}, M, f)$ denote a (continuous) flow on a metric space $M$. We shall use the convenient notation $f(t, x)=t x$ and $f(I \times A)=I A$, if $I \subset \mathbb{R}$ and $A \subset M$. The orbit of the point $x \in M$ will be denoted by $C(x)$. We recall that

$$
L^{+}(x)=\left\{y \in M: t_{n} x \rightarrow y \text { for some } t_{n} \rightarrow+\infty\right\}
$$

is the positive limit set of the point $x \in M$. The negative limit set $L^{-}(x)$ is defined analogously.

The orbit $C(x)$ is called Poisson stable if $x \in L^{+}(x) \cap L^{-}(x)$. A Poisson stable orbit is called strictly Poisson stable if it is not singular or periodic. A set $A \subset M$ is called minimal if it is non-empty, closed, invariant and has no proper subset with these properties. A minimal set is called trivial if it consists of only one orbit or is homeomorphic to the torus $T^{2}$.

We also recall that two flows $(\mathbb{R}, M, f)$ and $(\mathbb{R}, N, g)$ are topologically equivalent if there is a homeomorphism $h: M \rightarrow N$ sending orbits onto orbits preserving their time orientation. The reader is referred to [3] for the undefined terms used in the sequel. 
Proposition 2.1. Let $(\mathbb{R}, M, f)$ be a flow on a connected 2-manifold. If there exists a non-trivial compact minimal set $A$ which has an open invariant neighbourhood $V$ such that $L^{+}(y) \cap L^{-}(y) \neq \varnothing$ for every $y \in V$, then the flow is topologically equivalent to a Denjoy flow.

Proof. According to [2, Theorem 1.1], there is a connected, open, invariant neighbourhood $E$ of $A$ such that if $y \in E$ then $L^{+}(y) \cup L^{-}(y) \subset A \cup \partial E$ and $L^{+}(y)=A$ or $L^{-}(y)=A$. Therefore, our assumption and the minimality of $A$ imply that $L^{+}(y)=L^{-}(y)=A$ for every $y \in E$. If $E$ is not compact then the flow on $E$ can be extended to a continuous flow on the one-point-compactification $E \cup\{\infty\}$ of $E$ that fixes $\infty$. Since $L^{+}(y)=L^{-}(y)=A$ for every $y \in E$, the singleton $\{\infty\}$ is a saddle set, and by [3, Corollary 6.11] there exist points $y_{1}, y_{2} \in E$ such that $\infty \in L^{+}\left(y_{1}\right) \cap L^{-}\left(y_{2}\right)$, a contradiction. This shows that $E$ must be compact. Since $M$ is connected, $M=E$. Therefore $M$ is compact and the flow has no singular or periodic orbits. It follows from [6, Chapter I, Theorem 4.3.3] that the flow is topologically equivalent to a Denjoy flow on the torus.

Let $F$ denote the set of singular points of a given flow and $G$ the set of points whose orbits are homeomorphic to $\mathbb{R}$.

LEMMA 2.2. Let $(\mathbb{R}, M, f)$ be a flow on a closed 2-manifold which is not topologically equivalent to a Denjoy or irrational flow. Let $C(x)$ be a strictly Poisson stable orbit such that $\overline{C(x)}$ has an open, invariant neighbourhood $V$ such that $L^{+}(y)=L^{-}(y)$ for every $y \in V$. Then $G \cap \overline{C(x)} \neq \varnothing$ and the set $(G \cup F) \cap \overline{C(x)}$ is not closed.

Proof. Since $\overline{C(x)}$ is a strictly Poisson stable orbit closure, it does not contain periodic orbits. Assume that $G \cap \overline{C(x)}=\varnothing$. Then every orbit in $\overline{C(x)}$ is either strictly Poisson stable or singular, by our assumption. If the point $y \in C(x)$ is strictly Poisson stable, then $\overline{C(y)}=\overline{C(x)}$ by the Structure Theorem in [4]. Thus, necessarily $F \cap \overline{C(x)} \neq \varnothing$, because otherwise $\overline{C(x)}$ would be a non-trivial minimal set or a torus, and by Proposition 2.1 the flow would be topologically equivalent to a Denjoy or irrational flow. This means that the set $X=(M-F) \cap \overline{C(x)}$ is a noncompact, locally compact invariant subspace of $M$ carrying a minimal flow such that $L_{X}^{+}(y)=L_{X}^{-}(y)$ for every $y \in X$, where $L_{X}^{ \pm}$denotes limit set with respect to $X$. This, however, contradicts [7, Theorem 3]. So we have proved that $G \cap \overline{C(x)}$ is not empty. If now $(G \cup F) \cap \overline{C(x)}$ were closed, then the set $Y=(M-G \cup F) \cap \overline{C(x)}$ would be a non-compact, locally compact invariant subspace of $M$ carrying a minimal flow and we should have a contradiction as before.

Proof of Theorem 1.1. Let $(\mathbb{R}, M, f)$ be a flow on a closed 2-manifold $M$ with a strictly Poisson stable orbit $C(x)$ such that $\overline{C(x)}$ has an open invariant neighbourhood $V$ with the property $L^{+}(y)=L^{-}(y)$ for every $y \in V$. We shall assume that the flow is not topologically equivalent to a Denjoy or irrational flow, and arrive at a contradiction.

The connected component $N$ of $V-F \cap \overline{C(x)}$ containing $C(x)$ is a 2-manifold of finite genus. Hence, its end-point-compactification is a closed 2-manifold $N^{+}$. The 
ends of $N$ constitute a totally disconnected compact subset of $N^{+}$and correspond to boundary components of $N$ in $M$. The flow on $N$ can be extended to a continuous flow on $N^{+}$that fixes the ends [1, Section 2].

Lemma 2.2 implies that there exists a sequence $\left\{x_{n}: n \in \mathbb{N}\right\}$ of points of $G \cap \overline{C(x)}$ converging to some strictly Poisson stable point $y \in \overline{C(x)}$. By the Structure Theorem of [4] we have $\overline{C(x)}=\overline{C(y)}$ and we may therefore assume that $x=y$. Each point $x_{n}$ is non-wandering and thus $L^{+}\left(x_{n}\right)=L^{-}\left(x_{n}\right) \subset F \cap \overline{C(x)}$ by our assumption and [9, Proposition 5]. So we may assume that $C\left(x_{n}\right) \cap C\left(x_{m}\right)=\varnothing$ if $n \neq m$. The limit set $L^{+}\left(x_{n}\right)$ is a compact connected subset of the set $F \cap \overline{C(x)}$ and hence reduces to a point in $N^{+}$with respect to the extended flow. It follows that the set $C_{n}=\overline{C\left(x_{n}\right)}$ is a simple closed curve in $N^{+}$, where the closure is taken in $N^{+}$.

One can prove that the simple closed curves $C_{n}, n \in \mathbb{N}$, do not separate $N^{+}$for large enough values of $n$, using an argument similar to that used in the proof of Lemma 6 in [8]. Thus, we may assume that none of them separates $N^{+}$. If $C_{n} \cap C_{m}=\varnothing$ for infinitely many $n \neq m$, then there are three mutually different indices $i, j, k$ such that each pair of the simple closed curves $C_{i}, C_{f}, C_{k}$ bound an annulus [5, Appendix]. This means that one of them lies in the interior of the annulus bounded by the other two. Let $C_{i}, C_{j}$ bound the annulus $K$ and $C_{k} \subset \operatorname{int} K$. Then int $K$ is an open invariant neighbourhood of $C_{k}$ and therefore $C(x) \subset$ int $K$, because $x_{k} \in L^{+}(x)$. This, however, contradicts the Poincaré-Bendixson Theorem.

So we may assume that $C_{n} \cap C_{m} \neq \varnothing$ for all $n, m \in \mathbb{N}$. Hence, there is a singular point $z \in N^{+}$such that $C_{n} \cap C_{m}=\{z\}$ for $n \neq m$. Since the simple closed curve $C_{1}$ does not separate $N^{+}$, the set $N^{+}-C_{1}$ is a connected 2-manifold with one or two ends and its end-point-compactification $N_{1}$ is a closed 2-manifold of genus smaller than the genus of $N^{+}$. The flow on $N^{+}-C_{1}$ can be extended to a flow on $N_{1}$ that fixes the ends of $N^{+}-C_{1}$. Passing to a subsequence if necessary, we may assume that $L^{+}\left(x_{n}\right)=$ $L^{-}\left(x_{n}\right)=\{p\}$ or $L^{+}\left(x_{n}\right)=\{p\}$ and $L^{-}\left(x_{n}\right)=\{q\}$ in $N_{1}$ for all $n>1$, where $p, q$ are the ends of $N^{+}-C_{1}$. Let $C_{n}^{\prime}=\overline{C\left(x_{n}\right)}$ in the first case and $C_{n}^{\prime}=\overline{C\left(x_{n}\right)} \cup \overline{C\left(x_{2}\right)}, n>2$, in the second, where the closures are taken in $N_{1}$. Since $C(x)$ lies in $N^{+}-C_{1}$ and $x_{n} \rightarrow x$, in either case the simple closed curves $C_{n}^{\prime}, n>2$, do not separate $N_{1}$ for large values of $n$. Consequently, we may assume that $N_{1}-C_{3}^{\prime}$ is a connected 2-manifold with one or two ends, and its end-point-compactification is a closed 2-manifold of genus smaller than the genus of $N_{1}$. Continuing this process we arrive after a finite number of steps at a flow on a 2-manifold of genus zero carrying $C(x)$, which contradicts the Poincaré-Bendixson Theorem.

\section{References}

1. H. ABELS, 'Enden von Räumen mit eigentlichen Transformationsgruppen', Comment. Math. Helv. 47 (1972) $457-473$.

2. K. Athanassopoulos, 'The flow near non-trivial minimal sets on 2-manifolds', Math. Proc. Camb. Phil. Soc. 108 (1990) 569-573.

3. N. P. Bhatia, 'Attraction and non-saddle sets in dynamical systems', J. Differential Equations 8 (1970) 229-249.

4. C. Gutierrez, 'Smoothing continuous flows on 2-manifolds and recurrences', Ergodic Theory Dynamical Systems 6 (1986) 17-44.

5. C. L. Hagopian, 'Homogeneous continua in 2-manifolds', Topology Appl. 19 (1985) 157-163.

6. G. HECTOR and U. HirSCH, Introduction to the geometry of foliations, Part A (Vieweg, Braunschweig, 1981).

7. S. KoNo, 'Non-compact and non-trivial minimal sets of a locally compact flow', Tokyo J. Math. 5 (1982) 213-223.

8. E. Lima, 'Common singularities of commuting vector fields on 2-manifolds', Comment. Math. Helv. 39 (1964) 97-110. 
9. D. A. Neumman, 'Central sequences in flows on 2-manifolds of finite genus', Proc. Amer. Math. Soc. 61 (1976) 39-43.

Department of Mathematics

University of Crete

GR-71409 Iraklion

Crete

Greece 